\title{
On Sharing Knowledge And Fostering "Open Science"
}

\author{
by Erol Gelenbe, Guy Brasseur, Luc Chefneux, Véronique Dehant, Véronique \\ Halloin, Jean-Paul Haton, Michel Judkiewicz, Bernard Rentier, and Romain \\ Weikmans
}

\section{Editors' Introduction}

The crucial importance of science and technology and its accurate peer reviewed dissemination, has once again been demonstrated during the current pandemic. Thus the COVID-19 pandemic together with the inevitable energy transition required by climate change, lead us to consider the issue of scientific and technical communication, both for the written papers and proceedings that have largely moved online (but not always in open access), and the various types of seminars, workshops, and symposia that frequently involve air travel with substantial CO2 impact. Online meetings that have become recently very popular, as well as online repositories for publications, themselves have a significant CO2 - as well as environmental-impact, due to the massive use of electricity by information and communication technologies (ICT) and of the environmentally unfriendly manufacturing processes and decommissioning of ICT equipment. Presented is a broad overview of these aspects, and some recommendations regarding the future organization of scientific and technical communication, including: (1) peer-reviewed journals and proceedings with online open access; (2) the importance of face to face seminars and symposia, together with online meetings, to maintain the serendipity and importance of direct human contact while reducing the need for air travel; (3) the peer evaluation of research and of academic and research staff and its dependence on publications and their qualitative-rather than excessively quantitative-evaluation, where the concept of impact should include the usefulness of research to education, industry and society; (4) and the crucial role of ICT in all these aspects and the questions raised by the sustainability of ICT itself. 


\title{
On Sharing Knowledge And Fostering "Open Science"
}

\author{
by Erol Gelenbe, Guy Brasseur, Luc Chefneux, Véronique Dehant, Véronique \\ Halloin, Jean-Paul Haton, Michel Judkiewicz, Bernard Rentier, and Romain \\ Weikmans
}

The development of science and innovation involves different actors in society, each of which contributes in its way to the advancement of knowledge and thus to economic, social, and cultural development. These actors are of course scientists but also companies of all sizes, local authorities, cities, large national groups, and ultimately the largest possible number of citizens because science and innovation are everyone's business. The internet has sped up the dissemination of various types of information among this large group of actors and this inevitably obliges us to maintain an "open science," which allows us to quickly verify the validity of information that may be of importance to our wellbeing, safety, and health.

The recent COVID-19 crisis has made it clear that crisis management and unfounded statements or pressure from public opinion are no substitute for the requirements of a scientific approach, peer review, or structured, objective, and transparent research. It has illustrated the absolute need for a shift toward open science.

Thus, the results of research-not least publicly funded research-should generally, with some exceptions, be accessible and the resulting knowledge should remain freely available, without charge, to all citizens [1]. When this knowledge concerns legitimate issues of national security, it may be bound by rules regarding its confidentiality. Similarly, when knowledge leads directly to developments and inventions of commercial value, it may legitimately be exploited in compliance with intellectual property rules. Moreover, there is no denying the increasing use of results from one discipline in another one, and indeed from scientific and engineering disciplines to industry, business, and society; among many examples, chemistry and physics are extensively used in biology, engineering tools (such as robotics) are revolutionizing medical practice, while the use of computer technology affects practically all disciplines. This interaction, which has become commonplace, has increased the need for sharing and requires a rapid transfer of knowledge between actors and disciplines.

Traditionally, sharing knowledge through dissemination is based on work published in academic journals, on education and popularization, and on the organization of seminars, workshops and international conferences. To this, however, we must add ICT, which offers the ubiquitous means to store and disseminate knowledge. 
The COVID-19 pandemic, which suddenly confronted our societies with a problem that was all the more worrying precisely because of our lack of knowledge about it, combined with the questions raised for several years now by climate change, have led us to consider the issue of scientific communication and, in particular, the various types of meetings between researchers (seminars, symposia, conferences, digital social networks, etc.), some of which frequently involve substantial air travel. Indeed, the current situation has confronted us with both the absolute need to exchange knowledge and a communication means imposed by circumstances: teleconferences and giant "chats." While their existence is fully justified at present, what about their future?

It is clear to any researcher that human encounters are a powerful driving force in science. They help to inspire research, to avoid taking a dead-end road already tried by others, to launch collaborations between researchers working on similar or identical questions, to appraise each other, to unofficially validate an experimental approach, and to corroborate or validate risky hypotheses before undertaking cumbersome and/or lengthy procedures. These meetings also help establish contacts that will sometimes lead to a job, prepare a career transition, and set up postdoctoral stays for young researchers or a long-term stay at a renowned institution. This opportunity to meet must be preserved. But, this obvious fact aside, should we not ask more questions about the purpose and effectiveness of our trips in the context of our research activities?

\section{Major International Conferences and Workshops}

Traditional peer-reviewed publications fail to meet many specific but important needs and situations: a time frame and review cycles ("refereeing") that are sometimes far too long; and the possible "barrier" put up by peers who can be skeptical about a real innovation or brilliant concept. It may also be a waste of resources and time if the path followed in the publication submitted has already been unsuccessfully explored by others, and never published.

On the other hand, taking part, via oral presentations or "posters" or a mixture of the two ("PICOs"), in this diverse range of symposia and seminars that have become established in different disciplines over time-and the rapid means of communication they offer-makes it possible to test the field and interact in a timely manner. In addition, face-to-face discussion forums in small groups (workshops) allow for a very enriching exchange of ideas, which is difficult to replace with written correspondence or online meetings.

Nevertheless, the internet provides a practical alternative to some of these approaches by creating real dissemination and discussion forums that reduce the need for participants to travel and also facilitate the participation of researchers with limited travel budgets. Furthermore, a powerful accelerator compared to traditional publishing has evolved in the form of platforms - such as arXiv-which are open and free of charge (to authors and readers) and help disseminate prepublications quickly. While their open and immediate nature has clear 
advantages, it is certainly not free of drawbacks: Authors must be able to set a date very formally in order to be able to subsequently assert the anteriority of their discovery or invention, and must agree to expose themselves transparently to the criticism and recommendations of their peers, in the public eye rather than under the protection of editorial confidentiality.

It would therefore be in the interest of research and higher-education institutions to use reliable platforms that allow them to communicate easily at a distance, taking direct part in major international symposia. Universities can organize such events, just as they increasingly provide distance education and popularization courses.

The working group further recommends that all the preprints for these symposia submitted on reliable, permanent archiving platforms that are free of charge to researchers be used to supplement the presentations or abstracts at many conferences. This archiving could be accompanied by a process of refereeing and reviewing articles, leading to the validation of a publication under the supervision of a scientific editorial board, with the contribution of the academies who would agree, where appropriate, to take responsibility for it.

Furthermore, the working group strongly stresses the need for any public-research publication or prepublication to be submitted on platforms that must remain freely and openly accessible. This effort should be tied in with, on the one hand, the current open-science gains made in Germany (where works by German researchers are funded through the payment of a lump sum to several publishers) and, on the other hand, with the negotiations around Plan $S$ that aims at a similar result across Europe, in parallel with "open" archiving support for research work funded by the European Union.

The working group also proposes that higher-education institutions and research bodies, like all public and private organizations, should consider their policies regarding international travel and assess how they can offset the greenhouse gas emissions they cannot reduce.

Of course, the proliferation of discussion forums and archiving platforms requires investing in hardware and software, and this too leads to energy consumption and therefore to greenhouse gas emissions as well as the consumption of other natural resources, which have a serious impact on the environment $[2,3,4]$. It is therefore not a "miracle solution" and here too a careful analysis of the energy cost-efficiency ratio and environmental impact is required.

\section{Toward Scientific Communication Corridors In Europe}

Until now physical meetings in the field of research (seminars, symposia, conferences) were the norm, while virtual meetings were the exception. The COVID-19 crisis, however, has led to a rapid shift toward virtual meetings, the advantages and limitations of which everyone has been able to experience. One of these shortcomings is, by its very nature, the limiting of physical 
contact that is otherwise characteristic of human exchanges essential to research, thanks to their informal and spontaneous expression. Indeed, the virtues of coffee-break talks are well proven. We all know how much our ability to make progress in a research study "by chance" thanks to an unexpected discovery, or a sudden intuition, or serendipity-can benefit from such informal contacts and face-to-face meetings.

However, a growing awareness of the need for more sustainable development has for the past few years spurred reflections on the trips linked to scientific events and on the partial alternative offered by virtual meetings. If exchanges between people-in the field of research as in all sectors of human activity-are essential to the development of knowledge and innovations our societies need, we will probably not be able to do without a combination of face-to-face and virtual meetings. So we can envisage regular regional meetings and annual global meetings.

For regional meetings, better use should be made of the many European cities connected by high-speed rail corridors, which emit much fewer greenhouse gases than airplanes planes at an average ratio of 1 to 20 according to the European Environment Agency. In addition, trainswhether "normal," fast, or overnight ones-can be more convenient than planes if we take into account airport delays (access, security, and check-in and waiting times), provided that their timetables and frequency are adequate.

Thus, to facilitate face-to-face contacts as well as limit the carbon footprint of research trips as much as possible, the working group recommends that cycles of regional meetings be set up. In this case, and particularly in Europe, these meetings should be organized in places that are interesting hubs connected by fast rail corridors. Global plenaries could be organized according to a hybrid model whereby some participants would be physically present while others would attend by teleconference. There could thus be a hierarchy of meetings (local, regional, and global) that are clearly labeled to limit energy-intensive travel.

National and international academies and their networks (ALLEA, ICSU, Euro-CASE, CAETS, EASAC, etc.) could become more involved in this restructuring to help develop appropriate logistics and the dissemination of knowledge, both at a high level between peers, and in terms of smart dissemination and exchanges with citizens, NGOs, the public, and young citizens.

\section{Research Assessment}

Largely related to scientific communication, the assessment of researchers and research structures is an essential element of the research process in terms of both judging the quality of research and motivating researchers.

Assessment must be carried out by competent peers, in accordance with proper standards. Admittedly, any qualitative review by experts is necessarily tinged with a certain degree of 
subjectivity, which has often been blamed for the brake placed by peer review on the publication of ideas that are very innovative or break with current practice. We must be aware of this and take it into account since peer review remains, rightly so, the golden rule of the scientific world.

However, peer evaluation cannot be limited to calculating the various numerical indices, which are supposed to account for the productivity or creativity of a researcher. It must consist of a genuine assessment of the real value of the publications, their contribution to science, and of their contribution to the work. Indeed, a fair and balanced assessment should be based on: (a) the relevance, transparency, and fairness of the assessments, and (b) the competence, integrity, and diversity of the assessors. The impact of a researcher's work should also include, when appropriate, the usefulness of the research to education, industry, business, and society, as well as the adoption of its outcomes in practical settings, such as clinical practice (e.g. as in medicine) or products or business practice (e.g. as in management science).

Thus, the 2012 San Francisco Declaration on Research Assessment (DORA) and the 2015 Leiden Manifesto were intended to improve assessment practices in recruiting, promoting, or evaluating individual researchers. These documents, signed by numerous research and funding institutions, have alerted the research community to the misuse of certain bibliometric indicators, such as journal impact factors (JIF) that can be biased or manipulated to favor certain journals. Moreover, when it comes to the comparative assessment of researchers from different fields or even sub-specialties, it should take into account the diversity of disciplinary practices. Indeed, the total number of citations (using different sources) is often influenced by the number of articles and of co-authors, and may be of little relevance in some fields or in assessing the impact that a researcher may have on the practical and widespread usage of the concepts they may have developed or introduced.

The $\mathrm{H}$ index, a composite indicator supposed to simultaneously account for the number of a researcher's publications and their scientific impact, is appealingly simple but it depends on the number of publications, the JIF, the number of citations of a given publication, and thus on the number of years that have passed since it came out as well as its influence over a long period. Unfortunately, it suffers from the same defect as the JIF, while placing great importance on the number of publications, which tends to lead to the production of an abnormal quantity of articles with minimal added value. This aberration has also ended up saturating the peerreview process by eroding its quality, leading to a proliferation of commercially oriented journals that require authors to pay to be published.

Thus, the principles of open science require assessment that is based on multiple, essentially qualitative, criteria and is done by peers. This is a long and sometimes tedious task that in many cases requires more skill, effort, and analytical rigor than the mere addition of rather meaningless figures. It should not be carried out too often given its arduous nature: about once every five years seems reasonable. It should not be reduced to publication indicators even though publications remain an essential criterion for assessing dossiers. Indeed, an abundance 
of publications-and the number of co-authors per article-is not necessarily a reliable measure of the quality of a researcher or the importance of a research project, even if the number of publications compared to research seniority is low certainly calls for a vigilant examination of someone's real contribution, which may nevertheless prove to be significant.

In most cases, self-assessment can contribute to this more qualitative approach in the initial phase of the process. In a self-assessment dossier, those who are being assessed are asked to select their major works and, above all, to explain this selection: the scope and impact of a publication and their personal contribution. All works cited must be accessible in an open institutional archive. Researchers should be allowed to publish their manuscripts by uploading them to a prepublication server before submitting them for peer review. In this way, they can elicit comments from the scientific community, which they may take into account when making possible amendments. This is already the case in physics, mathematics, or economics.

It is important funding bodies and other stakeholders encourage this prepublication principle. And, once it has been accepted in its final form and as soon as the publisher allows it, any published document must be placed on an open-access institutional server.

We can see that open science can play a central role in research assessment. This fact must be taken into account by all actors: researchers, laboratories, research-funding bodies, and publishers. The situation is changing, slowly but in the right direction, and national academies should encourage this change.

Finally, assessment methods should be part of the doctoral training of all researchers. Indeed, they touch on the issue of scientific integrity, which is an essential part of the research profession.

Thus, the working group reaffirms the centrality of the peer assessment of researchers and research structures in the process of developing the quality of research, as well as in motivating researchers. However, these assessments should not be too frequent to maintain their importance: every five years would be reasonable. They should be carried out in accordance with international standards, in compliance with requirements of transparency, collegiality, and equal treatment, and by eminent peers from outside the structure being assessed or who have no professional relationship with the researcher whose dossier is being reviewed.

It is important to consider the specificities of each disciplinary field and to take into account criteria other than publication alone, such as a desire for openness (free access to publications as well as to research data, etc.), the results obtained in the doctoral training of young researchers, the time devoted to training researchers, or the contribution to the scientific community at a local, national, and international level. It is also important to pay attention to the wording of the questions put to the experts. A process that tries to obtain clear answers on specific points, often used by major American universities in the assessment of their faculty, 
could inspire the questions submitted to the assessors. When assessing a researcher, laboratory, organization, or research program it may be useful to look at the following:

- What are the main and important results obtained by the researcher (or laboratory, etc.) during the period under assessment, including details about the relevant publications?

- What new questions has the researcher (or laboratory, etc.) raised or worked on and published?

- What are the group projects to which the researcher (or the laboratory, etc.) has contributed or in which they have demonstrated intellectual or organizational "leadership"?

- What are the important services and contributions of the researcher (or laboratory, organization, or research program) to the international, national, institutional, and regional scientific community, and industry and business?

\section{The Carbon Footprint Of Academic Communication, Training, And Exchanges}

Universities and research bodies, just like society as a whole, are increasingly aware of the importance of climate change. They are thus striving to better understand and measure the carbon footprint of their activities, set their priorities in this area, and reduce their consumption of non-renewable energy and their $\mathrm{CO} 2$ emissions. Some institutions in Europe and elsewhere, such as the EPFL or the University of British Columbia, have even taken the initiative of publishing annual reports on this subject. These show that commuting and business travel account for more than 50 percent of the $\mathrm{CO} 2$ impact of these institutions. The muchencouraged globalization of universities, including education and academic exchanges, leads to much air travel that has a significant impact on $\mathrm{CO} 2$ emissions, often amounting to 30 percent or more of the total.

However, the difficulty of such assessments can easily be seen from a few examples. Thus, sending around 30 emails a day for a year results in the emission of around $600 \mathrm{~kg}$ of $\mathrm{CO} 2$, which is equivalent to around 50 percent of the annual emissions of an average citizen of India; while the purchase of $€ 5,000$ worth of ICT equipment represents the emission of around 2.8 tons of $\mathrm{CO} 2$ (without counting the carbon impact of its operation), which is greater than the 2.2 tons of $\mathrm{CO} 2$ per passenger of a two-way flight between Brussels and Beijing [5].

In these assessments, the many trips made by students to travel back and from their families when the latter live in a different country or city are rarely taken into account, even though students are an essential part of a university's community. Indeed, a European institution hosting 1,000 North or South American and Asian students expected to return home twice a year would add around 4,400 tons of $\mathrm{CO} 2$ to its carbon footprint; this is equivalent to the trips 
made by 420 "frequent-flyer" teacher-researchers, each of whom would fly twice a year to Asia, twice to the USA, and four times inside Europe.

Compared to other human activities such as leisure, the carbon footprint of researchers and scientific institutions may be very well justified. However, we should probably not overlook how crucially important is the example set by the scientific community, which makes the very recommendations aimed at drastically reducing greenhouse gas emissions. Therefore, the working group suggests research institutions and organizations, as all-public and private bodies, should publish annual reports on their energy consumption as well as an estimate of their $\mathrm{CO} 2$ footprint. The carbon footprint of universities can include commuting by staff and students, an estimate of the $\mathrm{CO} 2$ footprint of student and staff travel for academic purposes, and may also include information regarding the effect of an institution's internationalization and globalization objectives and strategy, as well as any carbon offsets.

\section{References}

[1] Rentier, B. Science ouverte. Le défi de la transparence. L'Académie en Poche 114 (2018). ISBN: 978-2-8031-0659-2.

[2] Pernici, B. et al. What IS can do for environmental sustainability: A report from CAize'11 panel on green and sustainable IS. Communications of the Association for Information Systems 30, 1 (2012).

[3] Gelenbe, E. and Caseau, Y. The impact of information technology on energy consumption and carbon emissions. Ubiquity Magazine. (June 2015). DOI: 10.1145/2755977

[4] Campana, M., Cuegniet, J., Schmitt, M. and Siben,C. Réduire la consommation énergétique du numérique. Rapport du Conseil général de l'économie. 2019.

[5] Moss. S. Pointless emails: they're not just irritating - they have a massive carbon footprint. The Guardian, Nov. 26, 2019.

\section{Biographies}

Erol Gelenbe, FACM, FIEEE, FIFIP graduated from METU (Ankara), received a Ph.D. from NYU, and the "Docteur d'état" from the Sorbonne. Active in research on computer and network performance evaluation, machine learning, and cybersecurity, he invented new mathematical models, such as the eponymous G-Networks, Random Neural Networks, and Energy Packet Networks. His team also built the first European system performance modeling package QNAP at INRIA, designed the FLEXSIM manufacturing system simulator, and patented the first voice- 
over-packet switch SYCOMORE. He received the 2008 ACM SIGMETRICS Award "for being the single individual who, over a span of 30 years, has made the greatest overall contribution to the field of computer system and network performance evaluation through original research, mentoring and doctoral training, creation and direction of world-class research groups, wideranging international collaboration, and professional service." Credited with having graduated more than 90 Ph.D.s, he also received the IET (UK) Olivier Lodge Medal (2010), the French National Academy of Sciences Grand Prix France Telecom (1996), the Mustafa Prize (2017), and honorary doctorates from the University of Rome, Italy, the University of Liège, and Bogazici University, Istanbul. Elected to chaired professorships at the University of Liège, University Paris-Saclay, University Paris-Descartes, the Polish Academy of Sciences, and named chairs at Duke University, University of Central Florida, and Imperial College London. Fellow of the French National Academy of Technologies, the Royal Academy of Belgium, the Science Academies of Hungary, Poland and Turkey, and Academia Europaea, he is a Chevalier de la Légion d'Honneur, and Commander of the Orders of Merit of France and Italy, and Chevalier des Palmes Académiques.

Guy P. Brasseur received degrees in physics, telecommunications, and electronics, and a Ph.D. from the Free University of Brussels Belgian Institute for Space Aeronomy, for advanced photochemistry and transport in the middle atmosphere. As a member of the Belgian House of Representatives, in 1988 he moved to NCAR in Boulder, CO, becoming Director of the Atmospheric Chemistry Division in 1990. Director of the Max Planck Institute for Meteorology in 2000, President of the Atmospheric Sciences Section, American Geophysical Union (20022004), lead author of the fourth Assessment Report (WG-1) of the International Panel for Climate Change (IPCC), his team won the 2007 Nobel Peace Prize jointly with Al Gore. During 2006-2009 as Associate Director of NCAR and Head of the Earth and Sun Systems Laboratory, he was named Founding Director of the Climate Service Center (CSC) in Hamburg in 2009. Current Member of the Max Planck Institute for Meteorology, Head of Environmental Modeling, his interests include global change, climate variability, chemistry-climate relations, biosphere-atmosphere interactions, climate change, stratospheric ozone depletion, global air pollution including tropospheric ozone, and solar-terrestrial relations. Awarded Doctor Honoris Causa by the Universities of Paris Pierre and Marie Curie, Oslo and Athens, he is a member of Academies of Hamburg, Brussels, and Oslo, and of Academia Europea.

Luc Cheffneux is currently a visiting professor at the University of Liege. Previously, he served as the President of the Section on Technology and Society of the Royal Academy of Sciences, Arts and Letters of Belgium, in 2019 and 2020. He has also been Director of Partnerships and European Affairs, coordinating the R\&D collaborations with external partners and the European Commission for ArcelorMittal Group, after having been Director of Open Innovation and Knowledge Management. Before that he was Director of the Scientific \& International Affairs of ArcelorMittal. He holds an engineering degree in physics engineering, and a Ph.D. from the University of Liege, Belgium. 
Véronique Dehant is a Belgian geodesist and geophysicist specializing in modeling the deformation of the Earth's interior in response to forcing from the Sun, Moon, and the Earth's rotation, and also studies Mercury, Venus, Mars, and icy satellites of the outer planets, at the Royal Observatory of Belgium, but also serves as an Extraordinary Professor at the Université Catholique de Louvain. She received all her degrees (B.S., M.S., and Ph.D.), in mathematics and physics from the Université Catholique de Louvain. Her research group was awarded for this work the 300,000 Euro Descartes Prize in 2003. Prof. Dehant is a co-investigator on the NASA RISE (Rotation and Interior Structure Experiment) and SEIS (Seismic Experiment for Interior Structure) projects, hosted by the InSight mission to Mars. The RISE team will use Doppler measurements to determine the rotation and position of Mars in space. This in turn provides information about the structure of the deep interior of Mars. Dehant's work has been widely recognized. In 2003 she received the European Geosciences Union geodesy prize, the VeningMeinesz Medal. She is a Fellow of the Royal Academy of Sciences, Arts and Letters of Belgium, a Fellow of the American Geophysical Union, and a foreign member of the French Academy of Sciences. In 2016 she received the Whitten medal from the American Geophysical Union for "outstanding achievement in research on the form and dynamics of the Earth and planets."

Véronique Halloin is the President of the European Science Foundation, the first woman president in its 47-year history. A former Professor of Chemical Engineering, since 2008 she is also General Secretary of the Fund for Scientific Research (F.R.S.-FNRS) of Belgium and has strong experience in research policy and research management. Under her leadership, the Belgian research funding agency was successful in reforming its evaluation procedures, adopting multi-annual strategic plans, and modernizing its research management tools in conformity with the highest standards in the field. Prior to that, as a civil and chemical engineer by education and Ph.D. holder in applied sciences, she served as Professor and Director of the Chemical Engineering Department of the School of Engineering at the Free University of Brussels (Université Libre de Bruxelles - ULB). Other leadership roles included acting as President of the School of Bioengineering and Vice-Rector of the ULB, responsible for research and development. An active member in several high-level international bodies and fora, such as the Science Europe, the European University Association, the CERN Council, and the Global Science Forum, she is also a past vice president of the European Science Foundation.

Jean-Paul Haton received the agrégé degree in physics at the Ecole Normale Supérieure de Saint-Cloud, and the docteur d'état in sciences from the University of Lorraine. He is presently Emeritus Professor in computer science at the Université de Lorraine in Nancy. He teaches various aspects of computer science and artificial intelligence. He has created in LORIA/INRIA the unit called "Pattern Recognition and Artificial Intelligence," which groups about 80 researchers who are now in different teams of the laboratory. His research field concerns the theoretical and applied aspects of perceptive and cognitive processes: knowledge-based systems, stochastic models, and neural nets, with applications in automatic speech recognition, signal interpretation, and decision-making. He is a senior member of Institut Universitaire de France (IUF) where he created the chair entitled "Digital Models of perceptive and cognitive processes." He has published more than 300 articles and books, and he has supervised or co- 
supervised about 80 Ph.D. theses. Haton graduated in 1993 from the French Institut des Hautes Études de Défense Nationale, IHEDN. He is a Fellow of the Royal Academy of Belgium and the Académie Lorraine des Sciences.

Michel Judkiewicz, a Fellow of the Royal Academy of Sciences, Arts and Letters of Belgium, is an expert on the processes and challenges related to innovation in business and industry. He has authored several articles and books in this area and is a frequently invited speaker at professional conferences and meetings. CEO of Silver Brains, he holds a master's degree combining electrical and mechanical engineering from the Université Libre de Bruxelles (ULB), and several business degrees from INSEAD and the University of Houston. A Visiting Professor at the University of Liège, he is also very active in engineering and social organizations. Past President of the Engineering Alumni of ULB, he has held various positions in industry and business, in Europe and the USA. He is the former Secretary General of EIRMA, the European Industry Research Management Association, an independent, not-for-profit organization with a European perspective of global management and organization of business research, development, and innovation, in 20 countries and 150 major companies operating in a wide range of sectors.

Bernard Rentier the former 60th Rector of the University of Liège, is a virologist and immunologist who received research training at the National Institute for Medical Research (Mill Hill, London) and the National Institutes of Health (Bethesda, Maryland), and then at the University of Liège. He is the author of more than 250 scientific publications. His scientific work has focused on influenza, measles, and chickenpox. He chaired the European branch of the Varicella-Zoster Virus Foundation (1991-2004) and the WAVE committee (Working against Varicella in Europe) and was vice-president of the European Federation of Biotechnology (1997-2004). He founded and chaired EOS (Enabling Open Scholarship), an international association for the promotion of free access to scientific publications (open access) in universities, and was awarded an honorary doctorate from the University of Quebec in Montreal in 2014 for his contributions to the promotion of the values of open access around the world. Also vice-president of the Belgian Federal Council for Science Policy, his book entitled Open Science, The Challenge of Transparency, which is online and free, won the Political Book Prize in Belgium. His awards include an NIH Fellowship, the Charles J. Ketelaer Prize of the Belgian League against Multiple Sclerosis, the Princess Joséphine-Charlotte Prize for the fight against viral infections of the nervous system and polio, and the honors of Commander of the Order of the Crown of Belgium, Grand Officer of the Order of Leopold II, and Chevalier des Palmes Académiques of France.

Romain Weikmans is a Postdoctoral Research Fellow of the Fund for Scientific Research (F.R.S.FNRS) at the Centre for Studies on Sustainable Development at the Université Libre de Bruxelles (ULB), where he received his Ph.D. in 2015. He was the top graduate of the master's program on the environment at the same university in 2011. His main research interest lies at the interface between climate change and foreign aid. During his Ph.D. he also held a visiting position at the University of Oxford. Currently also a Lecturer in the Faculty of Science (ULB) 
and at Sciences Po Lille, he is the Vice-Chair of the Working Group "Energy-Climate" of the Belgian Federal Council for Sustainable Development. He is a member of the Transdisciplinary Advisory Board of the Joint Programming Initiative "Connecting Climate Knowledge for Europe" (JPI Climate).

DOI: $10.1145 / 3462221$ 